\title{
BMJ open Comparison of cognitive decline between dementia with Lewy bodies and Alzheimer's disease: a cohort study
}

\author{
Zuzana Walker, ${ }^{1,2}$ Ian McKeith, ${ }^{3}$ Joanne Rodda, ${ }^{1}$ Tarik Qassem, ${ }^{2,4}$ Klaus Tatsch, ${ }^{5}$ \\ Jan Booij, ${ }^{6}$ Jacques Darcourt, ${ }^{7}$ John O'Brien ${ }^{3}$
}

To cite: Walker Z, McKeith I, Rodda J, et al. Comparison of cognitive decline between dementia with Lewy bodies and Alzheimer's disease: a cohort study. BMJ Open 2012;2: 000380

doi:10.1136/

bmjopen-2011-000380

- Prepublication history for this paper is available online. To view these files please visit the journal online (http:// dx.doi.org/10.1136/ bmjopen-2011-000380).

Author disclosure statements At the time of the study, ZW, JOB, IM, KT, JB and JD have received consultancy payments from GE Healthcare. JR has received funding for neuroimaging research from GE Healthcare. TQ has no disclosures.

Received 5 October 2011 Accepted 16 December 2011

This final article is available for use under the terms of the Creative Commons Attribution Non-Commercial 2.0 Licence; see http://bmjopen.bmj.com

\section{ABSTRACT}

Objectives: Dementia with Lewy bodies (DLB) accounts for $10 \%-15 \%$ of dementia cases at autopsy and has distinct clinical features associated with earlier institutionalisation and a higher level of carer distress than are seen in Alzheimer's disease (AD). At present, there is on-going debate as to whether DLB is associated with a more rapid cognitive decline than $A D$. An understanding of the rate of decline of cognitive and non-cognitive symptoms in DLB may help patients and carers to plan for the future.

Design: In this cohort study, the authors compared 100 $A D$ and 58 DLB subjects at baseline and at 12-month follow-up on cognitive and neuropsychiatric measures. Setting: Patients were recruited from 40 European centres.

Participants: Subjects with mild-moderate dementia. Diagnosis of DLB or AD required agreement between consensus panel clinical diagnosis and visual rating of 123I-FP-CIT (dopamine transporter) single photon emission computed tomography neuroimaging.

Outcome measures: The Cambridge Cognitive Examination including Mini-Mental State Examination and Neuropsychiatric Inventory (NPI).

Results: The AD and DLB groups did not differ at baseline in terms of age, gender, Clinical Dementia Rating score and use of cholinesterase inhibitors or memantine. NPI and NPI carer distress scores were statistically significantly higher for DLB subjects at baseline and at follow-up, and there were no differences between $A D$ and $D L B$ in cognitive scores at baseline or at follow-up. There was no significant difference in rate of progression of any of the variables analysed.

Conclusions: DLB subjects had more neuropsychiatric features at baseline and at follow-up than $A D$, but the authors did not find any statistically significant difference in rate of progression between the mild-moderate $A D$ and DLB groups on cognitive or neuropsychiatric measures over a 12-month follow-up period.

\section{INTRODUCTION}

end of article.

Correspondence to Dr Zuzana Walker; z.walker@ucl.ac.uk
Dementia with Lewy bodies (DLB) is the second most common form of neurodegenerative dementia and accounts for approximately $15 \%$ of cases of dementia at

\section{ARTICLE SUMMARY}

Article focus

- Dementia with Lewy bodies (DLB) has distinct neuropsychiatric features

- At present, we do not know whether the poorer prognosis of DLB is due to a more rapid cognitive decline compared with Alzheimer's disease (AD)

\section{Key messages}

- In this fairly large cohort of patients with DLB and $A D$, while there was no difference in level of cognitive impairment (Cambridge Cognitive Examination (CAMCOG) score) at baseline and at 12-month follow-up, DLB patients had significantly higher Neuropsychiatric Inventory (NPI) and NPI carer distress scores both at baseline and at 12-month follow-up.

- Therefore, the worse prognosis of DLB is likely to be mediated by neuropsychiatric or other symptoms and not only by cognitive decline

Strengths and limitations of this study

- Inclusion of high number of subjects from 40 European clinical centres

- Well-characterised cases with both consensus panel clinical diagnosis (three clinical experts) and dopaminergic transporter single photon emission computed tomography imaging

- No autopsy data were available and therefore it is possible that more rapid cognitive decline may be present in pure DLB

- Only 1 year of follow-up

- There was higher attrition rate (no-follow-up assessment) in the DLB group, and DLB patients that did not return for follow-up were more impaired than $A D$ patients.

autopsy. ${ }^{1}$ It is characterised by the central feature of a progressive dementia accompanied by one or more core features of fluctuations in cognition, visual hallucinations and spontaneous features of parkinsonism. ${ }^{2}$

Awareness of the rate of cognitive decline and also of non-cognitive symptoms can help carers and patients to adjust and plan appropriate lifestyle changes and to make 
arrangements for the future. This frequently involves making difficult decisions regarding treatment of psychiatric and motor symptoms and utilisation of limited resources available for patients with dementia.

Since its recognition as a neurodegenerative disorder, a body of research has focused on the differentiation of DLB from other dementias, in particular Alzheimer's disease $(\mathrm{AD})$, in terms of both cross-sectional and longitudinal clinical factors. In addition to the clinical syndrome described in the consensus diagnostic criteria, DLB is associated with higher levels of behavioural disturbance and care giver distress, lower quality of life and greater demand on resources when compared with $\mathrm{AD} .{ }^{3}$ Despite these findings, there is uncertainty within the literature regarding progression and survival in DLB compared with $\mathrm{AD}$. Studies have shown survival in DLB to be either comparable to ${ }^{5}$ or shorter ${ }^{6}$ than in AD. No differences in decline on global measures (eg, Clinical Dementia Rating (CDR)) have been reported. ${ }^{7}$ Studies of the progression of cognitive impairment have generally reported a similar ${ }^{7} 8$ or steeper ${ }^{5}$ rate of decline in DLB when compared with AD. An exception to this was a study by Stavitsky et $a{ }^{\ominus}$ where $\mathrm{AD}$ patients had a steeper decline on cognitive and behavioural measures, although DLB patients had been more impaired at baseline.

Comparisons of longitudinal outcomes between DLB and $\mathrm{AD}$ to date have generally needed to trade off diagnostic accuracy against prospective study design. Autopsy studies have the benefit of definitive diagnosis but are usually dependent on retrospective analysis of clinical data. Studies using clinical diagnosis often have the advantage of prospective study design but at the expense of diagnostic accuracy. Overall, the majority of studies of the 1996 clinical consensus criteria for DLB ${ }^{10}$ have identified high specificity, with lower estimates of sensitivity. While one study identified $83 \%$ sensitivity and $95 \%$ specificity, estimates of sensitivity from other studies have been as low as $23 \%^{11} 12$ with reports of specificity ranging from $8 \%$ to $100 \%$; the most frequent misdiagnosis of DLB is as $\mathrm{AD} .{ }^{13}$

The development of ${ }^{123}$ I-FP-CIT single photon emission computed tomography (SPECT) now allows visualisation of striatal dopamine transporter, and consequentially dopaminergic degeneration in vivo, and differentiates between $\mathrm{AD}$ and DLB with a sensitivity and specificity of $78 \%-88 \%$ and $94 \%-100 \%$, respectively. ${ }^{14}$ An abnormal visual rating on ${ }^{123}$ I-FP-CIT SPECT was incorporated into the most recent revision of the consensus diagnostic criteria. ${ }^{2}$ In the present study, our aim was to compare decline in cognitive, behavioural and global measures over a 12-month period in a prospectively followed cohort of subjects with either $\mathrm{AD}$ or DLB confirmed by consensus panel clinical diagnosis and normal (for $\mathrm{AD}$ ) and abnormal (for DLB) ${ }^{123}$ I-FP-CIT SPECT imaging.

\section{METHODS}

Data were collected as part of a phase 3 multicentre imaging study whose methodology is described in detail elsewhere. ${ }^{15} 16$ In brief, patients were aged 55-90 years and met the criteria for dementia detailed in DSM-IV and fulfilled at least one of the following: consensus criteria for DLB ${ }^{10}$ NINCDS-ADRDA criteria for probable or possible $\mathrm{AD}^{17}$ or NINDS/AIREN criteria for probable or possible vascular dementia. ${ }^{18}$ A Mini-Mental State Examination (MMSE) score at baseline of $\geq 10$ was required to ensure patients could complete assessments. ${ }^{19}$ Patients with dementia who developed parkinsonism >1 year before the onset of dementia were deemed to have Parkinson's disease with dementia and were not included. ${ }^{10}$ Those with structural imaging findings indicative of infarction in the region of the basal ganglia, including the internal capsule, were excluded. Use of medication known or suspected to interact with striatal binding of ${ }^{123}$ I-FP-CIT was not permitted. ${ }^{20}$

The study was done in accordance with the current revision of the Declaration of Helsinki and the Good Clinical Practice: Consolidated Guideline approved by the International Conference on Harmonisation and applicable to national and local laws and regulations. At every participating site, the study protocol and all amendments were approved by an institutional review board or independent ethics committee. All patients and care givers gave written informed consent.

Following inclusion in the initial study, participants were invited for clinical and neuropsychological reassessment at 12 months.

Clinical diagnosis at baseline, as previously reported, was established by an independent consensus panel of three specialist clinicians, who were provided with a patient profile compiled from quality-assured clinical data from the on-site investigators' case record forms and copies of on-site original source data. ${ }^{15}$ The same panel reconvened to consider the baseline and the 12-month follow-up data to arrive at a second and final consensus diagnosis. This final consensus diagnosis was used to derive the cohort for the present study.

The following were undertaken at baseline and followup: MMSE, Unified Parkinson's Disease Rating Scale III (motor section), ${ }^{21}$ modified Hoehn and Yahr staging, ${ }^{22}$ clinical assessment of cognitive fluctuation scale ${ }^{23}$ the Cambridge Cognitive Examination-revised version (CAMCOG-R) ${ }^{24}$ Neuropsychiatric Inventory with care giver input (NPI-D), ${ }^{25}$ visual object and space perception (VOSP) battery ${ }^{26}$ and CDR. ${ }^{27}$ The Cornell Scale for Depression in Dementia ${ }^{28}$ and the investigator's estimation of the patient's IQ level were completed at baseline but not at follow-up. Results of MRI and CT scans and the on-site investigators' clinical diagnosis before imaging were also available. The consensus panel did not have access at any stage to ${ }^{123}$ I-FP-CIT SPECT findings and was unaware of the patients' identities and the names of the centres and the investigators. Before any cases were diagnosed, the consensus panel was asked to diagnose 10 patients (separate to the study) for whom autopsy diagnosis was independently available. There 
was $100 \%$ concordance between the diagnoses made by the panel and at autopsy. ${ }^{15}$ Individual panel members reviewed each study case, including the baseline consensus panel diagnosis and all subsequent information, before meeting to agree a final clinical diagnosis of probable DLB, possible DLB or non-DLB dementia. Patients in the non-DLB category were further allocated to probable or possible $\mathrm{AD}$, probable or possible vascular dementia or other.

Within a few weeks of the baseline clinical diagnosis, SPECT images were acquired on a 2 or 3 headed $\gamma$ camera (SPECT system) 3-6 h after a single intravenous injection of 111-185 MBq of ${ }^{123}$ I-FP-CIT $^{29}$ (DaTSCAN, the radiotracer was supplied by GE Healthcare Buchler GmbH \& Co. KG, Munich, Germany). See McKeith et $a l^{15}$ for details. Subjects underwent standard thyroid blocking. SPECT imaging was not repeated at follow-up. As previously described, three nuclear medicine physicians assessed scans, blind to diagnosis, using a 4-point scale (0, normal uptake; 1 , unilateral putamen loss; 2, bilateral putamen loss and 3 , virtually absent uptake $)^{15}$; we used only the dichotomous division of normal (0) versus abnormal (1-3) images for analysis. For the present study, we combined the three independent reads and recorded the result of the scan as normal or abnormal if there was agreement between two or more raters.
For the purposes of the present study, we included only patients with complete data sets from both baseline (T1) and 1-year follow-up (T2) assessments and with reliable images from the baseline ${ }^{123}$ I-FP-CIT SPECT session $(n=225)$. These patients were divided into two diagnostic groups (AD and DLB). Inclusion criteria for the $\mathrm{AD}$ group were a consensus diagnosis of possible or probable $\mathrm{AD}$ at 12-month follow-up in addition to a negative (normal) ${ }^{123}$ I-FP-CIT SPECT read $(n=100)$. To be included in the DLB group, patients were required to have a consensus diagnosis of probable DLB at 12month follow-up and to have a positive (abnormal) ${ }^{123}$ I-FP-CIT SPECT image read $(n=58)$. Patients with a dementia diagnosis other than possible or probable $\mathrm{AD}$ or probable DLB were excluded $(n=50)$, see flowchart in figure 1.

\section{STATISTICAL ANALYSIS}

Data were analysed using SPSS V.18. We compared baseline and 12-month follow-up data and change over time for the $\mathrm{AD}$ and DLB groups. $\chi^{2}$ tests were used to assess differences between the diagnostic groups (AD and DLB) with respect to gender and medication use at baseline. For normally distributed variables, $t$ tests were used for between-group comparisons of baseline and follow-up variables. Mann-Whitney $\mathrm{U}$ tests were used for non-normally distributed baseline and follow-up data.
Figure 1 Flowchart of subjects included in the study. The flowchart details patients who completed both baseline and follow-up assessments. Twentyfive patients with a diagnosis of dementia with Lewy bodies (DLB) at baseline and 19 patients with a diagnosis of Alzheimer's disease $(A D)$ at baseline did not return for follow-up and are therefore not included in the flowchart.

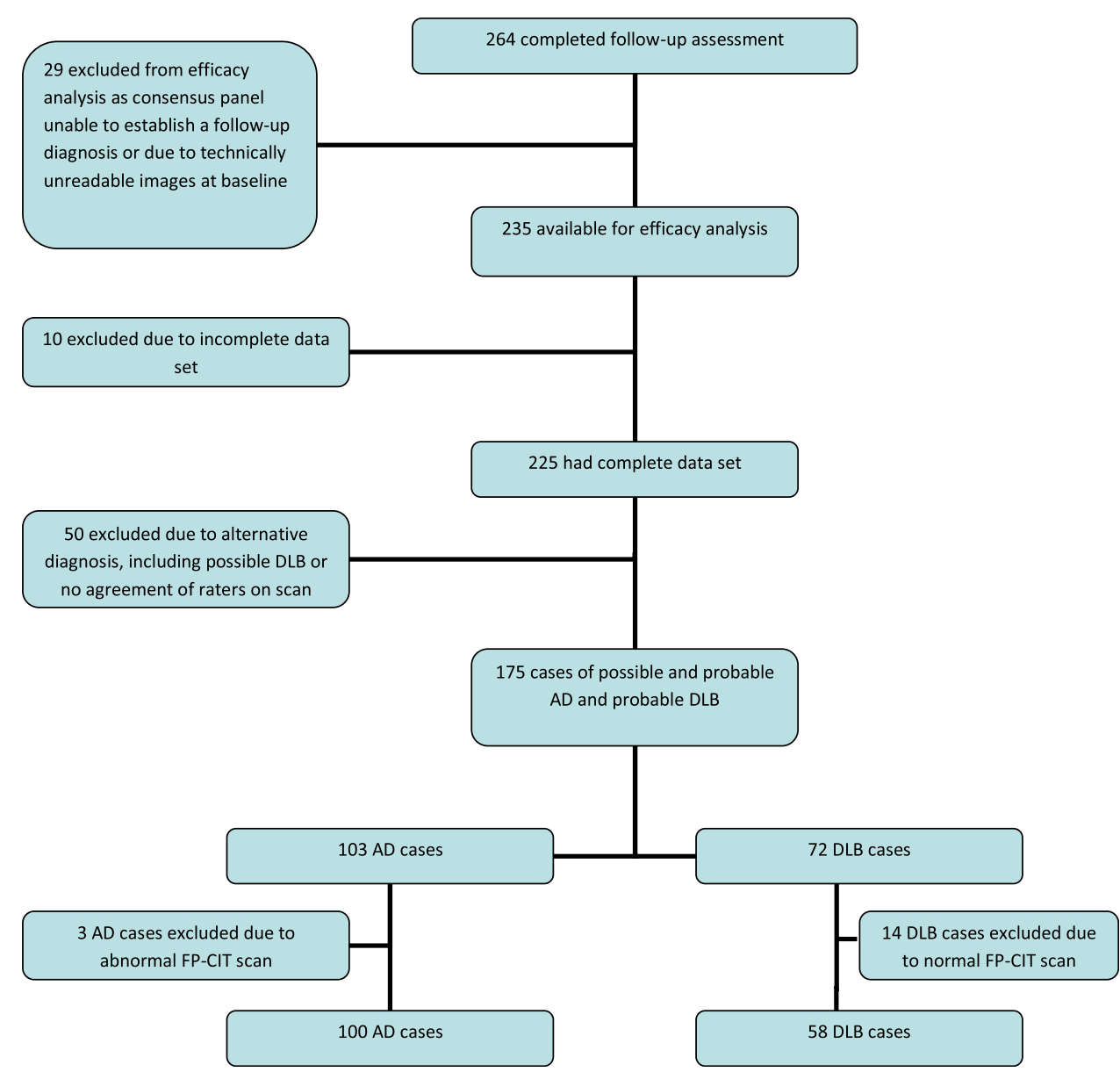


Repeated measures analysis of variance was used for analysis of group $\times$ time interactions (comparison of change in variables over time in each group). General Linear Models with fixed effect were used to adjust for the difference in NPI scores and the scores on the Cornell Scale for Depression in Dementia at baseline.

\section{RESULTS}

Baseline and follow-up data for the DLB and AD groups are presented in table 1 . The groups did not differ in terms of age or gender. There were no between-group baseline differences in terms of CDR or use of cholinesterase inhibitors or memantine. The DLB group had a statistically significantly higher mean depression score at baseline and higher scores on the NPI, NPI carer distress and clinical assessment of cognitive fluctuation scales at both baseline and 12-month follow-up $(p<0.001)$. There were no differences between the groups on cognitive scores at either baseline or follow- up. No significant differences in change in scores between baseline and 12-month follow-up for any of the variables analysed were identified. Results of analysis did not change when data were stratified according to gender. There was also no statistically significant difference between DLB and $\mathrm{AD}$ in the change of CAMCOG score after adjustment for scores on NPI and Cornell Scale for Depression scores. There were numerically greater changes (more decline) between baseline and follow-up on cognition, fluctuation and on measures of neuropsychiatric symptoms in the DLB patients but this did not reach statistical significance. The lack of a significant difference on the cognitive performance between the DLB and AD groups is unlikely to be due to lack of power, therefore type II error, as the effect size of that difference was very small $(0.02-0.07)$ and only a sample size of 1685 subjects would have shown a significant difference.

A higher number of DLB patients (25) compared with $\mathrm{AD}$ patients (19) did not return for a follow-up visit. DLB

Table 1 Baseline characteristics and scores on clinical scales and cognitive testing at baseline and at 12-month follow-up and change in scores between time points for Alzheimer's disease (AD) and dementia with Lewy bodies (DLB) groups

\begin{tabular}{|c|c|c|c|}
\hline & $A D(n=100)$ & DLB $(n=58)$ & $\mathbf{p}$ \\
\hline \multicolumn{4}{|l|}{ Gender (M:F) } \\
\hline $\mathrm{M}$ & $48(48 \%)$ & $37(64 \%)$ & \multirow[t]{2}{*}{0.06} \\
\hline $\mathrm{F}$ & $52(52 \%)$ & $21(36 \%)$ & \\
\hline Age in years at ${ }^{123}$ I-FP-CIT SPECT session & $74.9(7.3)$ & $74.2(6.1)$ & 0.53 \\
\hline Cornell Scale for Depression in Dementia (baseline) & $3.8(3.3)$ & $6.6(3.4)$ & $<0.001$ \\
\hline Cholinesterase inhibitor & $82(82 \%)$ & $45(76 \%)$ & 0.50 \\
\hline Memantine & $9(9 \%)$ & $2(3 \%)$ & 0.19 \\
\hline Neuroleptic medication & $4(4 \%)$ & $9(16 \%)$ & 0.01 \\
\hline Clinical Dementia Rating (baseline) & $1.2(0.69)$ & $1.3(0.66)$ & 0.3 \\
\hline \multicolumn{4}{|l|}{ MMSE score (SD) } \\
\hline Baseline & $21.5(4.5)$ & $21.4(3.9)$ & 0.85 \\
\hline Follow-up & $19.0(6.2)$ & $18.5(6.0)$ & 0.65 \\
\hline Change & $2.6(4.0)$ & $3.1(4.3)$ & 0.40 \\
\hline \multicolumn{4}{|l|}{ CAMCOG score (SD) } \\
\hline Baseline & $66.3(15.6)$ & $66.0(13.5)$ & 0.89 \\
\hline Follow-up & $59.5(20.3)$ & $56.3(19.7)$ & 0.35 \\
\hline Change & $7.5(10.6)$ & $9.0(11.9)$ & 0.429 \\
\hline \multicolumn{4}{|l|}{ NPI score (SD) } \\
\hline Baseline & $9.7(10.3)$ & $19.8(14.6)$ & $<0.001$ \\
\hline Follow-up & $12.3(13.3)$ & $24.2(17.4)$ & $<0.001$ \\
\hline Change & $2.5(14.8)$ & $3.8(15.5)$ & 0.59 \\
\hline \multicolumn{4}{|l|}{ NPI-carer score (SD) } \\
\hline Baseline & $5.8(6.0)$ & $10.8(8.0)$ & $<0.001$ \\
\hline Follow-up & $5.8(5.7)$ & $11.8(8.6)$ & $<0.001$ \\
\hline Change & $-0.05(6.4)$ & $0.8(7.1)$ & 0.44 \\
\hline \multicolumn{4}{|l|}{ Fluctuations } \\
\hline Baseline & $0.6(2.1)$ & $6.0(4.5)$ & $<0.001$ \\
\hline Follow-up & $0.4(1.7)$ & $6.9(4.1)$ & $<0.001$ \\
\hline Change & $-0.2(2.4)$ & $0.8(4.1)$ & 0.07 \\
\hline \multicolumn{4}{|l|}{ CAMCOG executive function } \\
\hline Baseline & $11.9(5.2)$ & $11.1(4.7)$ & 0.33 \\
\hline Follow-up & $10.5(5.4)$ & $9.4(5.0)$ & 0.24 \\
\hline Change & $1.5(3.6)$ & $1.4(4.0)$ & 0.83 \\
\hline
\end{tabular}

Data are presented as mean (SD) or number (\%). $p=p$ Values (analysis of variance).

CAMCOG, Cambridge Cognitive Examination; MMSE, Mini-Mental State Examination; NPI, Neuropsychiatric Inventory. 
patients lost to follow-up were significantly more cognitively impaired than $\mathrm{AD}$ patients lost to follow-up at baseline on MMSE (score 17.3 vs 22.2), CAMCOG (score 53.1 vs 66.7) and executive function (score 8.4 vs 13.3). Since these patients lost to follow-up were not given a final diagnosis, they were not included in the main analysis.

\section{DISCUSSION}

In a prospectively followed sample of patients with clinical consensus panel and dopamine transporter SPECT confirmed diagnosis of $\mathrm{AD}$ or DLB, cross-sectional assessments identified expected between-group similarities and differences in cognitive scores and clinical scales in addition to a higher level of carer distress relating to the symptoms of DLB patients. We identified no differences in rate of progression of cognitive or neuropsychiatric variables over a 12-month follow-up period. Our inclusion of only patients whose consensus clinical diagnosis was in keeping with neuroimaging results make it likely that diagnostic accuracy was very high.

The $\mathrm{AD}$ and DLB groups were well matched in terms of age and degree of cognitive impairment at baseline. The finding that patients with DLB had higher scores on the NPI, clinician assessment of fluctuation and Cornell Scale for Depression in dementia were expected given the recognised diagnostic criteria. ${ }^{2}$

NPI score was higher at both time points in DLB, despite similar cognitive and baseline CDR scores; this was associated with higher levels of care giver distress and is in keeping with other published data. ${ }^{40}$ Severity of neuropsychiatric symptoms in $\mathrm{AD}^{31}$ and $\mathrm{DLB}^{32}$ has been shown to be a predictor of both care giver distress and nursing home placement. Care giver distress has also been shown to be an independent risk factor for nursing home placement in dementia. ${ }^{33}$ It is possible that the shorter time to nursing home placement that has been reported in DLB compared with $\mathrm{AD}^{34}$ is related to neuropsychiatric symptoms and associated care giver distress. Not all studies are consistent, however, and marginal $^{6}$ or no differences ${ }^{8}$ in time to placement have also been reported. Furthermore, costs of care in DLB and AD have been shown to correlate with impairments in activities of daily living (ADLs) and not NPI scores. ${ }^{4}$

Severity of neuropsychiatric symptomatology, and hallucinations in particular, has also been associated with steeper decline in cognitive scores and increased risk of mortality and institutionalisation in $\mathrm{AD}$, independent of antipsychotic drug use. ${ }^{35} 36$ These studies have lacked autopsy confirmation of diagnosis, and it is possible that the AD groups included individuals with undiagnosed DLB, who are more likely to experience hallucinations. We are not aware of any published data related to the impact of neuropsychiatric symptom severity on illness progression and survival in DLB.

We did not identify any between-group differences in change over time of any of the variables examined, that is, NPI, fluctuations and cognitive performance. It is possible that the lack of detectable difference in decline of NPI and fluctuation scores over time is related to the already high scores at baseline in DLB. The majority of studies of the rate of cognitive decline in DLB versus $\mathrm{AD}$ have also reported no differences, for example, ${ }^{7} 8$ although the earliest reports were of more rapid decline in cognition in DLB, ${ }^{37}$ as were more recent studies. ${ }^{5}$ Several studies have reported relatively preserved cognitive scores, particularly in recall, before death in DLB compared with $\mathrm{AD} .{ }^{5}$ As mentioned in 'Results', patients diagnosed as having DLB at baseline who were lost to follow-up were significantly more cognitively impaired than patients diagnosed as having $\mathrm{AD}$ at baseline who were lost to follow-up. These patients were not included in the final analysis, as the cohort analysed was derived from the final consensus diagnosis made at follow-up. Thus, although patients lost to follow-up appeared to differ cognitively depending on diagnosis and this could have affected the study's results, their diagnoses were not made at the same time point as for the patients included in the final cohort. Reviewing the characteristics of patients lost to follow-up must therefore be done tentatively, as their diagnoses were subject to change.

It has been suggested that DLB may be associated with a more rapid decline in global measures of dementia severity or measures of ADLs while cognitive performance is relatively preserved. However, no significant differences in change in CDR score over time between the $\mathrm{DLB}$ and $\mathrm{AD}$ groups have yet been identified. ${ }^{7} \mathrm{We}$ did not examine performance on ADLs. Cross-sectional assessments of activities of living have reported higher levels of impairment in DLB than in $\mathrm{AD},{ }^{9}{ }^{30}$ which may be related to extrapyramidal motor symptoms. ${ }^{38}$ Longitudinal data, however, suggest no difference or a marginal difference in rate of decline of ADLs between $\mathrm{AD}$ and DLB. ${ }^{9}$

While ours and the majority of studies do not support the idea of a more rapid decline in cognition in DLB, the available literature is split more evenly between findings of either similar or shorter survival in DLB compared with $\mathrm{AD}$. One possibility is that reports of worse outcomes in DLB are related to increased frequency of antipsychotic use as a result of greater severity of neuropsychiatric symptoms. While more DLB than $\mathrm{AD}$ participants were prescribed neuroleptics in the present study, no differences in rate of progression were identified. Previous studies of cognitive decline in AD and DLB that have presented data on neuroleptic prescribing did not report any differences between the groups in use of these medications. ${ }^{8}{ }^{39}$ In terms of survival, both early ${ }^{40}$ and more recent ${ }^{6} 8$ studies have reported shorter survival in DLB versus $\mathrm{AD}$, despite likely changes in neuroleptic prescribing over this time as a result of better understanding of the potentially harmful effects in both DLB ${ }^{2} 10$ and dementia as a whole. It therefore seems unlikely that reported 
differences in survival between DLB and AD could be entirely accounted for by antipsychotic use.

The literature surrounding the differences in longitudinal outcomes in DLB and AD is therefore not easy to interpret. Overall, studies report outcomes in DLB that are either no different from or worse than in AD. Some of the difficulties involved in interpreting and comparing these findings are the differences in study design, use of clinical rather than pathological diagnosis, differing pathological definitions and retrospective analysis of clinical data. In addition, studies often rely on relative's reports on the onset of dementia, or use as baseline the time of referral, diagnosis or entry into the study. None of these methods necessarily identify equivalent disease stages and these difficulties highlight the complexity of the task of comparing the rate of decline between two disorders with different clinical phenotypes. In DLB, episodic memory is relatively spared in the early stages, but the presence of attentional and visuospatial impairments, visual hallucinations or movement disorder might be more disabling. Comparisons between $\mathrm{AD}$ and $\mathrm{DLB}$ are therefore not straightforward, and it is hard to define what is an 'equivalent' disease stage. The picture is further complicated by the frequent overlap of $\mathrm{AD}$ and $\mathrm{DLB}$ neuropathology and the insidious onset of both of these conditions.

Our study would have been improved by a longer duration of follow-up and a more detailed breakdown of cognitive, behavioural and clinical measures. Furthermore, patients' ability to carry out ADLs was not measured, and this can be a useful marker of disease severity and progression. Exclusion of individuals with severe dementia and higher attrition (not returning for follow-up visit) of DLB cases with more severe cognitive impairment precluded detection of differences in progression that are present only in later disease stages. Larger cohorts of patients who could be stratified by stages of severity of dementia are needed to examine this possibility. The DLB group had a higher mean depression score at baseline and more patients took a neuroleptic. Both neuroleptics and antidepressants have been shown to have detrimental effect on patients with dementia and could lead to faster progression but this did not seem to be the case over the duration of 1 year. Without autopsy diagnosis, we were not able to differentiate patients with pure and combined pathology.

In conclusion, on global cognitive measures, we did not find any difference in rate of progression between the mild-moderate AD and DLB groups over a 1-year period of observation. Cognitive decline is only one aspect of dementia and other impairments may in fact be more important and disabling.

\section{Author affiliations}

${ }^{1}$ Research Department of Mental Health Sciences, University College London, Bloomsbury Campus, London, UK

${ }^{2}$ North Essex Partnership Foundation NHS Trust, Essex, UK

${ }^{3}$ Institute for Ageing and Health, Newcastle University, Newcastle upon Tyne, UK
${ }^{4}$ Institute of Psychiatry, Ain Shams University, Cairo, Egypt

${ }^{5}$ Department of Nuclear Medicine, Städtisches Klinikum Karlsruhe, Karlsruhe, Germany

${ }^{6}$ Department of Nuclear Medicine, Academic Medical Centre, Amsterdam, Netherlands

${ }^{7}$ Centre Anoine Lacassagne Department of Nuclear Medicine, Medical Faculty, University of Nice Sophia-Antipolis, Nice, France

Contributors ZW: involved in conception and design of the study as well as being a member of the consensus panel, contributed to statistical analysis and interpretation, co-wrote initial draft of the manuscript and prepared the final version of the manuscript; IM: involved in conception and design of the study as well as being a member of the consensus panel, contributed to and approved the final version of the manuscript; JR: involved in conception and design of the study, contributed to data analysis and interpretation, co-wrote initial and subsequent drafts of the manuscript, contributed to and approved the final version of the manuscript; TQ: involved in conception and design of the study, data processing, analysis and interpretation, contributed to and approved the final version of the manuscript; KT: involved in conception and design of the study, performed the visual analysis of the SPECT data, contributed to and approved the final version of the manuscript; JB: involved in conception and design of the study, performed the visual analysis of the SPECT data, contributed to and approved the final version of the manuscript; JD: involved in conception and design of the study, performed the visual analysis of the SPECT data, contributed to and approved the final version of the manuscript; JOB: involved in conception and design of the study as well as being a member of the consensus panel, contributed to and approved the final version of the manuscript.

Funding The data collection was sponsored by GE Healthcare who made data available for further analysis for the present study.

Competing interests None.

Ethics approval Ethical approval in 20 countries obtained (various).

Provenance and peer review Not commissioned; externally peer reviewed.

Data sharing statement The data used in this study were supplied by, and remain the property of, GE Healthcare. Hence, these are not available for sharing

\section{REFERENCES}

1. Holmes C, Cairns N, Lantos $\mathrm{P}$, et al. Validity of current clinical criteria for Alzheimer's disease, vascular dementia and dementia with Lewy bodies. Br J Psychiatry 1999;174:45-50.

2. McKeith IG, Dickson DW, Lowe J, et al. Diagnosis and management of dementia with Lewy bodies: third report of the DLB Consortium. Neurology 2005;65:1863-72.

3. Bostrom F, Jonsson L, Minthon L, et al. Patients with dementia with Lewy bodies have more impaired quality of life than patients with Alzheimer disease. Alzheimer Dis Assoc Disord 2007;21:150-4

4. Bostrom F, Jonsson L, Minthon L, et al. Patients with Lewy body dementia use more resources than those with Alzheimer's disease. Int J Geriatr Psychiatry 2007;22:713-19.

5. Nelson PT, Kryscio RJ, Jicha GA, et al. Relative preservation of MMSE scores in autopsy-proven dementia with Lewy bodies. Neurology 2009;73:1127-33.

6. Williams MM, Xiong C, Morris JC, et al. Survival and mortality differences between dementia with Lewy bodies vs Alzheimer disease. Neurology 2006;67:1935-41.

7. Johnson DK, Morris JC, Galvin JE. Verbal and visuospatial deficits in dementia with Lewy bodies. Neurology 2005;65:1232-8.

8. Hanyu H, Sato T, Hirao K, et al. Differences in clinical course between dementia with Lewy bodies and Alzheimer's disease. Eur J Neurol 2009;16:212-17.

9. Stavitsky K, Brickman AM, Scarmeas N, et al. The progression of cognition, psychiatric symptoms, and functional abilities in dementia with Lewy bodies and Alzheimer disease. Arch Neurol 2006;63:1450-6.

10. McKeith IG, Galasko D, Kosaka K, et al. Consensus guidelines for the clinical and pathologic diagnosis of dementia with Lewy bodies (DLB): report of the consortium on DLB international workshop. Neurology 1996;47:1113-24.

11. Lopez OL, Becker JT, Kaufer DI, et al. Research evaluation and prospective diagnosis of dementia with Lewy bodies. Arch Neurol 2002;59:43-6. 
12. O'Brien JT, Colloby S, Fenwick J, et al. Dopamine transporter loss visualized with FP-CIT SPECT in the differential diagnosis of dementia with Lewy bodies. Arch Neurol 2004;61:919-25.

13. Barker WW, Luis CA, Kashuba A, et al. Relative frequencies of Alzheimer disease, Lewy body, vascular and frontotemporal dementia, and hippocampal sclerosis in the State of Florida Brain Bank. Alzheimer Dis Assoc Disord 2002;16:203-12.

14. Walker Z, Jaros E, Walker RW, et al. Dementia with Lewy bodies: a comparison of clinical diagnosis, FP-CIT SPECT imaging and autopsy. J Neurol Neurosurg Psychiatry 2007;78:1176-81.

15. McKeith I, O'Brien JT, Walker Z, et al. Sensitivity and specificity of dopamine transporter imaging with 123I-FP-CIT SPECT in dementia with Lewy bodies: a phase III, multicentre study. Lancet Neurol 2007;6:305-13.

16. O'Brien JT, McKeith IG, Walker Z, et al. Diagnostic accuracy of 123I-FP-CIT SPECT in possible dementia with Lewy bodies. $\mathrm{Br} \mathrm{J}$ Psychiatry 2009;194:34-9.

17. McKhann G, Drachman D, Folstein M, et al. Clinical diagnosis of Alzheimer's disease: report of the NINCDS-ADRDA Work Group under the auspices of Department of Health and Human Services Task Force on Alzheimer's Disease. Neurology 1984;34:939-44.

18. Roman GC, Tatemichi TK, Erkinjuntti T, et al. Vascular dementia: diagnostic criteria for research studies. Report of the NINDS-AIREN International Workshop. Neurology 1993;43:250-60.

19. Folstein MF, Folstein SE, McHugh PR. "Mini-mental state". A practical method for grading the cognitive state of patients for the clinician. J Psychiatr Res 1975;12:189-98.

20. Booij J, Kemp P. Dopamine transporter imaging with [(123)I]FP-CIT SPECT: potential effects of drugs. Eur J Nucl Med Mol Imaging 2008;35:424-38.

21. Fahn S, Elton RL. Unified Parkinson's disease rating Scale. In: Fahn S, Marsden DC, Goldstein M, et al, eds. Recent Developments in Parkinson's Disease. McMillan 1987:153-63.

22. Hoehn MM, Yahr MD. Parkinsonism: onset, progression, and mortality. 1967 Neurology 1998;50:318.

23. Walker MP, Ayre GA, Cummings JL, et al. The clinician assessment of fluctuation and the one day fluctuation assessment scale. Two methods to assess fluctuating confusion in dementia. $\mathrm{Br} J$ Psychiatry 2000;177:252-6.

24. Huppert FA, Brayne C, Gill C, et al. CAMCOG-a concise neuropsychological test to assist dementia diagnosis: sociodemographic determinants in an elderly population sample. Br J Clin Psychol 1995;34:529-41.

25. Cummings JL, Mega M, Gray K, et al. The Neuropsychiatric inventory: comprehensive assessment of psychopathology in dementia. Neurology 1994;44:2308-14.
26. Warrington EK, James M. A new test of object decision: 2D silhouettes featuring a minimal view. Cortex 1991;27:370-83.

27. Hughes CP, Berg L, Danziger WL, et al. A new clinical scale for the staging of dementia. Br J Psychiatry 1982;140:566-72.

28. Alexopoulos GS, Abrams RC, Young RC, et al. Cornell scale for depression in dementia. Biol Psychiatry 1988;23:271-84.

29. Booij J, Hemelaar TG, Speelman JD, et al. One-day protocol for imaging of the nigrostriatal dopaminergic pathway in Parkinson's disease by [123l]FPCIT SPECT. J Nucl Med 1999;40:753-61.

30. Ricci M, Guidoni SV, Sepe-Monti M, et al. Clinical findings, functional abilities and caregiver distress in the early stage of dementia with Lewy bodies (DLB) and Alzheimer's disease (AD). Arch Gerontol Geriatr 2009;49:e101-4.

31. Rocca P, Leotta D, Liffredo C, et al. Neuropsychiatric symptoms underlying caregiver stress and insight in Alzheimer's Disease. Dement Geriatr Cogn Disord 2010;30:57-63.

32. Leggett AN, Zarit S, Taylor A, et al. Stress and burden among caregivers of patients with Lewy body dementia. Gerontologist 2011:51:76-85

33. Gaugler JE, Yu F, Krichbaum K, et al. Predictors of nursing home admission for persons with dementia. Med Care 2009; $47: 191-8$

34. Rongve A, Skogseth R, Aarsland D. Risk of nursing home placement in dementia with Lewy bodies and Alzheimer's dementia. Ref Type: Abstract. Alzheimer's Demen 2010;5(Suppl 4):79.

35. Wilson RS, Tang Y, Aggarwal NT, et al. Hallucinations, cognitive decline, and death in Alzheimer's disease. Neuroepidemiology 2006;26:68-75

36. Scarmeas N, Brandt J, Albert $\mathrm{M}$, et al. Delusions and hallucinations are associated with worse outcome in Alzheimer disease. Arch Neurol 2005;62:1601-8.

37. Olichney JM, Galasko D, Salmon DP, et al. Cognitive decline is faster in Lewy body variant than in Alzheimer's disease. Neurology 1998;51:351-7.

38. McKeith IG, Rowan E, Askew K, et al. More severe functional impairment in dementia with Lewy bodies than Alzheimer disease is related to extrapyramidal motor dysfunction. Am J Geriatr Psychiatry 2006;14:582-8.

39. Weiner MF, Risser RC, Cullum CM, et al. Alzheimer's disease and its Lewy body variant: a clinical analysis of postmortem verified cases. Am J Psychiatry 1996;153:1269-73.

40. Ballard C, Holmes C, McKeith I, et al. Psychiatric morbidity in dementia with Lewy bodies: a prospective clinical and neuropathological comparative study with Alzheimer's disease. Am J Psychiatry 1999;156:1039-45. 